\title{
RELAÇÃO ENTRE O TEOR DE UMIDADE E A CONSTANTE DIELÉTRICA DE ESPÉCIES DE MADEIRA DA AMAZÔNIA DURANTE O PROCESSO DE SECAGEM ${ }^{1}$
}

\author{
Matheus Couto Crisóstomo ${ }^{2 *}$, Fernando Nunes Gouveia ${ }^{3}$ e Alexandre Florian da Costa ${ }^{4}$
}

\footnotetext{
${ }^{1}$ Recebido em 24.10.2014 aceito para publicação em 20.11.2015.

${ }^{2}$ Universidade de Brasília, Graduado em Engenharia Florestal, Brasília, Distrito Federal - Brasil. E-mail: <matheuscc50@hotmail.com>

${ }^{3}$ Serviço Florestal Brasileiro, Laboratório de Produtos Florestais, Brasília, Distrito Federal - Brasil. E-mail: <fernando.gouveia@florestal.gov.br>.

${ }^{4}$ Universidade de Brasília, Departamento de Engenharia Florestal, Faculdade de Tecnologia, Brasília, Distrito Federal - Brasil. E-mail: <lucate@unb.br>

${ }^{*}$ Autor para correspondência.
}

\begin{abstract}
RESUMO - O objetivo deste estudo foi determinar a relação entre constante dielétrica e teor de umidade de seis espécies de madeira, por meio da construção de um capacitor. O material utilizado constituiuse de madeiras da Amazônia não comercializadas, mas com alta abundância e dominância na Floresta Nacional de Tapajós, Estado do Pará, como: Albizia duckeana (fava-paricá), Brosimum sp. (amapá-doce), Chamaecrista scleroxylon (pau-santo), Qualea dinizii (mandioqueira-rosa), Trattinnickia burserifolia (amescla) e Swartzia laurifolia (gombeira). Os corpos de prova para determinação da constante dielétrica foram confeccionados com as dimensões de $4,5 \times 4,5 \times 1 \mathrm{~cm}$, definidas por testes preliminares realizados no Laboratório de Produtos Florestais (LPF). Para a coleta dos valores de capacitância, foi também construído um capacitor, utilizando multímetro digital, fios de cobre e placas de alumínio, sendo as medições realizadas em oito ocasiões, com intervalos de $1 \mathrm{~h}$ cada, durante o processo de secagem, que se iniciou na condição de massa saturada até atingir massa constante. A partir dos dados, foram ajustadas as curvas de regressão entre os valores de teor de umidade e constante dielétrica da madeira, as quais apresentaram forte correlação com as duas variáveis, mostrando a influência direta e proporcional do teor de umidade sobre a constante dielétrica. Em razão da inexistência de relação direta entre os dados de massa específica e constante dielétrica das espécies estudadas, sugere-se o desenvolvimento de novos estudos para melhor explicar a relação entre essas duas grandezas.
\end{abstract}

Palavras-chave: Madeiras amazônicas; Capacitância; Material dielétrico.

\section{RELATION BETWEEN HUMIDITY VALUES AND THE DIELECTRIC CONSTANT OF SPECIES OF AMAZONIAN FOREST WOODS DURING THE DRYING PROCESS}

\begin{abstract}
The objective of this study was to determine the relation between dielectric constant and the humidity of six species of wood, by the construction of a capacitor. The material used was non commercialized Amazonian wood, but with high abundance and domination in the National Forest of Tapajos - State of Para, such as: Albizia duckeana (fava-paricá), Brosimum sp. (amapá-doce), Chamaecrista scleroxylon (pau-santo), Qualea dinizii (mandioqueira-rosa), Trattinnickia burserifolia (amescla) and Swartzia laurifolia (gombeira). The samples used to determinate the dielectric constant were made with the dimensions of: $4.5 \times 4.5 \times 1 \mathrm{~cm}$, defined by preliminary tests made at Forestry Products Laboratory (LPF). The values of capacitance were collected by the construction of a capacitor using a digital multimeter, copper wires, and aluminum plates; and the values were collected eight times with intervals of one hour, during the drying process, starting at saturated mass to constant mass. Therefore, with the collected data the regression curves were established between humidity values and the dielectric constant of wood, which
\end{abstract}


showed a strong correlation between the two variables, showing the direct and proportional influence of the humidity over the dielectric constant. Due to the inexistence of direct relation between specific mass and dielectric constant of the studied species, we suggest new studies to explain the relation between those characteristics.

Keywords: Amazonian wood; Capacitance; Dielectric material.

\section{INTRODUÇ̃̃o}

Sabe-se que o teor de umidade, isto é, a quantidade de água presente na madeira, exerce forte influência sobre as propriedades e respostas que esse material apresenta em seus diversos possíveis usos. O aumento do teor de umidade da madeira afeta, principalmente, as propriedades mecânicas, que reduzem a sua resistência aos esforços mecânicos e alteram a sua estabilidade dimensional, bem como suas propriedades elétricas, reduzindo o seu caráter isolante, além da sua resistência ao ataque de organismos xilófagos, conforme constatado por vários autores.

Decorre desse fato que uma secagem adequada contribui para o aumento da qualidade do produto final, de modo a atender a padrões exigidos pela indústria e pelos órgãos certificadores. Portanto, torna-se imprescindível conhecer o teor de umidade da madeira, e, para tal, o método mais utilizado é o método gravimétrico, que consiste na secagem de amostra com teor de umidade desconhecido até atingir massa constante. Entretanto, é possível encontrar diversos equipamentos no mercado que permitem a mensuração dessa variável de maneira portátil, acessível e rápida. Esses equipamentos são caracterizados por fazer a leitura do teor de umidade da madeira a partir de suas propriedades elétricas, considerando-se o tipo capacitivo, ou resistivo de acordo com o princípio utilizado para mensuração do teor de umidade. Todavia, a maioria dos aparelhos disponíveis no mercado para medição do teor de umidade é importado, ou seja, equipamentos desenvolvidos e calibrados com espécies oriundas do Hemisfério Norte, geralmente madeiras que apresentam características e comportamentos bem diferentes das madeiras tropicais. Além disso, Norimoto e Yamada etal. (1971) constataram que é possível observar valores distintos da propriedade dielétrica nos diferentes sentidos de orientação de corte da madeira, justificando a necessidade de se realizarem estudos que procuram preencher essa lacuna.

Nesse contexto, o objetivo deste trabalho foi correlacionar a constante dielétrica e o teor de umidade de seis espécies de madeiras amazônicas durante o processo de secagem por meio de curvas de regressão.

\section{MATERIAL E MÉTODOS}

Este trabalho foi realizado nas dependências do Laboratório de Produtos Florestais - LPF, do Serviço Florestal Brasileiro (SFB). As amostras foram confeccionadas utilizando seis espécies de madeiras oriundas da Floresta Nacional do Tapajós, no Município de Belterra, no Estado do Pará, sendo elas: Albizia duckeana L. Rico (fava-paricá), Brosimum sp. (amapádoce), Chamaecrista scleroxylon (Ducke) H.S. Irwin \& Barneby (pau-santo), Qualea dinizii Ducke (mandioqueira-rosa), Trattinnickia burserifolia Mart. (amescla) e Swartzia laurifolia Benth. (gombeira).

\subsection{Confecção dos corpos de prova}

As amostras foram obtidas a partir do abate de cinco árvores por espécie, com diâmetro mínimo de 45 $\mathrm{cm}$, sendo essas desdobradas em duas toras, cada uma com $180 \mathrm{~cm}$ de comprimento, sendo uma da base e outra do fuste da árvore. As toras foram divididas em toretes e pranchas e enviadas a cada uma das sete áreas de pesquisa do LPF. Das peças destinadas à Área de Secagem da Madeira foram escolhidas, aleatoriamente, tábuas que foram desdobradas em amostras com as seguintes dimensões: 45 x 45 x 10 mm (comprimento x largura $\mathrm{x}$ espessura), totalizando 180 , com 30 amostras por espécie.

\subsection{Secagem das amostras}

As amostras foram acondicionadas em um tanque com água até atingirem a saturação. Cada espécie foi submetida a um processo de secagem com duração de $8 \mathrm{~h}$, a $100^{\circ} \mathrm{C}$. As 30 amostras de cada espécie foram divididas em seis grupos, contendo cinco amostras cada. Esses grupos foram colocados na estufa em intervalos de 10 min entre eles. Após $1 \mathrm{~h}$ de permanência em estufa, o primeiro grupo foi retirado, e sua massa e capacitância foram medidas e, imediatamente, recolocado na estufa para continuar com o processo de secagem. Dez minutos após a retirada do primeiro grupo, o segundo conjunto de amostras foi retirado da estufa e seus dados de massa e capacitância, medidos. Assim, essa operação foi repetida para todos os grupos, mantendo o intervalo de 1 h entre uma medição e outra até completarem 8 $\mathrm{h}$ de permanência em estufa. 
As amostras foram reduzidas em seis grupos para minimizar a adsorção de água durante a coleta de dados. O processo de secagem foi realizado em estufa de laboratório com circulação forçada de ar tipo MARCONI modelo 032/1. A pesagem das amostras foi realizada utilizando balança analítica tipo METTLER modelo PM6100, com precisão de 0,01 g. A perda de massa foi mensurada ao longo da secagem, para que fosse possível calcular o teor de umidade (equação 1) e relacionar os valores de constante dielétrica, calculada em razão da capacitância coletada.

$$
T U-\frac{(m i-m f)}{m i} \times 100
$$

em que $T U$ é o teor de umidade (\%), mi a massa inicial (g) e MF a massa final (g).

\subsection{Coleta dos valores de capacitância}

Para a coleta dos valores de capacitância, foi necessária a construção de um capacitor. Para isso, foi utilizado um medidor de componentes eletrônicos MINIPA Modelo MX - 901, duas placas de alumínio de 45 x 45 x $1 \mathrm{~mm}$, dois fios de cobre com 2,5 mm de espessura, uma garra de plástico, fita isolante para fixar os fios de cobre nas placas e o material dielétrico, sendo todas as amostras de madeira estudadas feitas conforme modelo da Figura 1. Em razão da baixa ordem de grandeza dos valores de capacitância coletados, foi necessário ajustar a escala utilizada no aparelho para picofarads $\left(10^{-12} \mathrm{~F}\right)$.

\subsection{Análise dos Dados Coletados}

A análise dos dados foi realizada com o auxílio do Software Curve Expert 1.3, o qual permite o ajuste de curvas de regressão quadráticas de cada espécie, que podem ser aplicadas para modelagem de curvas de secagem. Matematicamente, é expressa de acordo com a equação 2 .

$$
T U-a+b k+c k^{2}
$$

em que TU é o teor de umidade (\%), $\mathrm{k}$ a constante dielétrica e a, b e c os coeficientes estimados por regressão.

\section{RESULTADOS}

Os valores de massa específica básica das espécies trabalhadas foram obtidas anteriormente pelo Laboratório de Engenharia do LPF/SFB, por meio de ensaios de

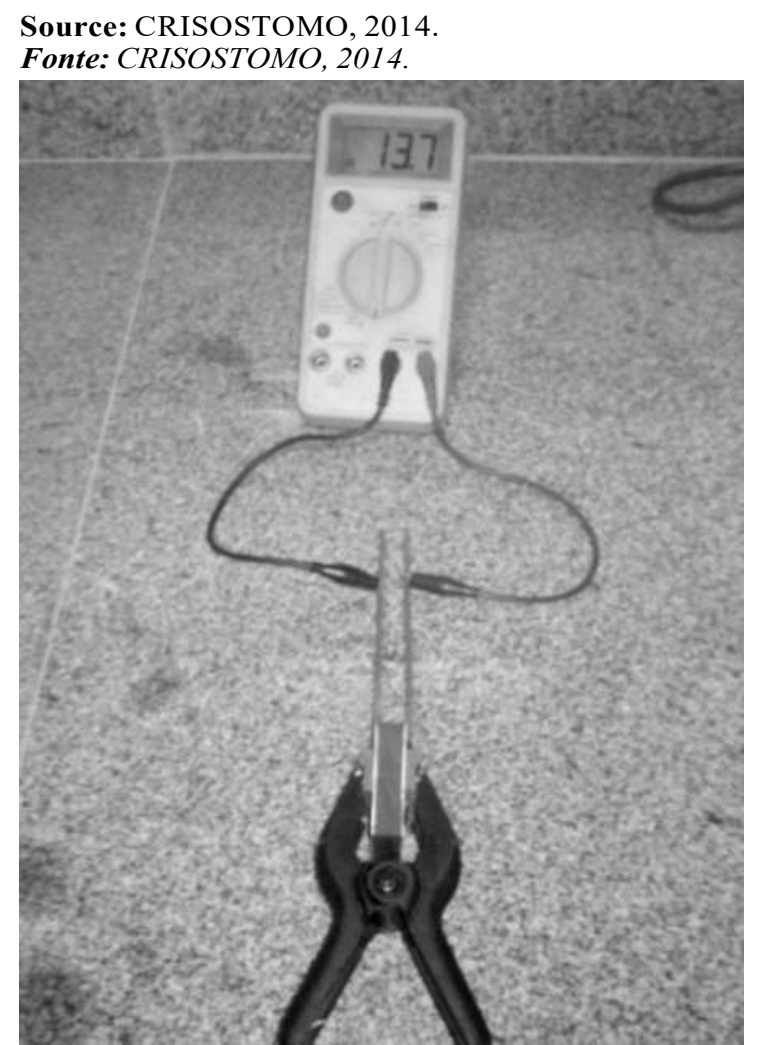

Figura 1 - Capacitor construído para coleta da capacitância. Figure 1 - Capacitor built for capacitance collection.

acordo com padrões estabelecidos na literatura, sendo obtidos à partir da média de 30 corpos de prova em condição saturada. Dessa forma, os valores de massa específica básica $\left(\mathrm{g} / \mathrm{cm}^{3}\right)$ para as espécies estudadas são: Albizia duckeana $\left(0,41 \mathrm{~g} / \mathrm{cm}^{3}\right)$, Trattinnickia burserifolia $\left(0,44 \mathrm{~g} / \mathrm{cm}^{3}\right)$, Brosimum $\mathrm{sp} .\left(0,54 \mathrm{~g} / \mathrm{cm}^{3}\right)$ Qualea dinizii $\left(0,57 \mathrm{~g} / \mathrm{cm}^{3}\right)$, Chamaecrista scleroxylon $\left(0,89 \mathrm{~g} / \mathrm{cm}^{3}\right)$, e Swartzia laurifolia $\left(1,03 \mathrm{~g} / \mathrm{cm}^{3}\right)$.

Portanto, no presente trabalho foram utilizadas espécies classificadas como de baixa, média e alta massa específica de acordo com o sistema de classificação das espécies com base na massa específica básica utilizada pelo Instituto de Pesquisas Tecnológicas (IPT).

\subsection{Valores Médios de Constante Dielétrica em Função do Teor de Umidade das Espécies}

A Tabela 1 apresenta os valores médios do Teor de Umidade (\%) e Constante Dielétrica para cada medição (oito no total) ao longo do processo de secagem. Dessa forma, as espécies foram listadas levando-se em consideração a massa específica em ordem decrescente

Revista Árvore, Viçosa-MG, v.40, n.1, p.181-187, 2016 
no sentido vertical, para ser possível observar o comportamento da constante dielétrica e como a massa específica e o teor de umidade são alterados.

É possível observar que houve redução abrupta no valor da constante dielétrica da primeira medição, quando se encontrava na condição saturada; na segunda, quando foi retirada após $1 \mathrm{~h}$ da estufa, para todas as espécies estudadas e, da segunda medição em diante, a alteração da constante dielétrica ocorreu de maneira menos acentuada. Portanto, os dados da primeira medição, se utilizados, interfeririam negativamente no ajuste da curva de regressão. Por isso, o ajuste foi realizado a partir da segunda medição.

\subsection{Curvas e Equações Ajustadas}

Como resultado da análise dos dados, foi possível o ajuste de curvas de regressão e de equações relacionando os valores coletados de teor de umidade e constante dielétrica. Por apresentar elevado coeficiente de determinação $\left(\mathrm{R}^{2}\right)$, valor que indica quanto da variação total é comum aos elementos que constituem os pares analisados, as equações permitissem o cálculo do teor de umidade da madeira a partir da sua propriedade dielétrica com alto grau de confiabilidade. A Tabela 2 apresenta a equação ajustada e o coeficiente de determinação $\left(\mathrm{R}^{2}\right)$ para as espécies estudadas.

A Figura 2 apresenta as curvas de regressão ajustadas para cada espécie, organizadas de acordo com a sua massa específica. Dessa forma, é possível observar que em todas as espécies há relação diretamente proporcional entre o teor de umidade e a constante dielétrica, de modo que esta última tende a reduzir o seu valor quando ocorre a retirada de água, comportamento que pode ser justificado pela menor capacidade de armazenamento de eletricidade dentro da amostra. Entretanto, o que diferencia cada curva de regressão é a massa específica, característica física de cada espécie que relaciona a quantidade de madeira que há em determinado volume. Todas as curvas de regressão apresentaram Coeficientes de determinação $\left(\mathrm{R}^{2}\right)$ elevados, demonstrando a alta correlação entre as variáveis analisadas.

A Tabela 3 apresenta os valores máximos e mínimos de constante dielétrica, teor de umidade e variação observada.

Brosimum sp., de massa específica média $(0,54$ $\mathrm{g} / \mathrm{cm}^{3}$ ), apresentou o maior (606) e o menor valor (11) de constante dielétrica, induzindo a existência de outros

Tabela 1 - Valores médios de teor de umidade e constante dielétrica.

Table 1 -Medium values of moisture content and dielectric constant.

\begin{tabular}{|c|c|c|c|c|c|c|c|c|}
\hline Espécies/Medições & 1 & 2 & 3 & 4 & 5 & 6 & 7 & 8 \\
\hline Swartzia laurifolia & $67.127,57$ & 322,06 & 268,41 & 236,33 & 206,06 & 197,83 & 192,56 & 56,99 \\
\hline Teor de Umidade (\%) & 35,90 & 24,71 & 21,14 & 18,56 & 16,64 & 15,16 & 13,86 & 0,49 \\
\hline Chamaecristas cleroxylon & $47.983,54$ & 173,12 & 125,37 & 94,40 & 71,29 & 60,23 & 48,00 & 56,71 \\
\hline Teor de Umidade (\%) & 48,81 & 26,33 & 17,15 & 11,40 & 7,70 & 5,55 & 4,20 & 1,40 \\
\hline Qualea dinizii & $153.020,58$ & 241,22 & 173,70 & 137,12 & 105,28 & 74,06 & 53,38 & 26,96 \\
\hline Teor de Umidade (\%) & 103,59 & 64,27 & 43,76 & 29,60 & 19,38 & 11,86 & 6,62 & 0,00 \\
\hline Brosimum sp. & $220.213,99$ & 372,16 & 139,72 & 94,75 & 72,53 & 48,87 & 35,85 & 18,44 \\
\hline Teor de Umidade (\%) & 117,45 & 69,97 & 45,74 & 29,61 & 18,04 & 10,05 & 5,05 & 0,95 \\
\hline Trattinnickia burserifolia & $145.629,63$ & 386,95 & 182,96 & 133,84 & 98,19 & 72,84 & 45,07 & 35,46 \\
\hline Teor de Umidade (\%) & 138,94 & 85,98 & 58,44 & 38,82 & 24,72 & 14,53 & 0,08 & 0,00 \\
\hline Albizia duckeana & $236.493,83$ & 401,98 & 160,76 & 84,40 & 70,88 & 58,93 & 48,58 & 27,24 \\
\hline Teor de Umidade (\%) & 181,30 & 121,64 & 89,89 & 65,95 & 47,19 & 33,86 & 23,29 & 1,74 \\
\hline
\end{tabular}

Tabela 2 - Equação ajustada e coeficiente de determinação $\left(\mathrm{R}^{2}\right)$.

Table 2 -Adjusted equations and coefficient of determination $\left(R^{2}\right)$.

\begin{tabular}{lll}
\hline Espécie & \multicolumn{1}{c}{ Equação } & \\
\hline Albizia duckeana & $-6,36+0,76 \mathrm{k}-0,0010 \mathrm{k}^{2}$ & 0,9502 \\
Brosimum sp. & $-6,49+0,40 \mathrm{k}-0,0005 \mathrm{k}^{2}$ & 0,9775 \\
Chamaecristas cleroxylon & $-8,66+0,25 \mathrm{k}-0,0003 \mathrm{k}^{2}$ & 0,8937 \\
Qualea dinizii & $-7,66+0,27 \mathrm{k}-0,00008 \mathrm{k}^{2}$ & 0,9818 \\
Trattinnickia burserifolia & $-14,49+0,45 \mathrm{k}-0,0004 \mathrm{k}^{2}$ & \\
Swartzia laurifolia & $-6,44+0,13 \mathrm{k}-0,0001 \mathrm{k}^{2}$ & 0,9775 \\
\hline
\end{tabular}

Revista Árvore, Viçosa-MG, v.40, n.1, p.181-187, 2016 
fatores determinantes para justificar a variação apresentada pela espécie.

Albizia duckeana e Trattinnickia burserifolia, de massa específica baixa $\left(0,41 \mathrm{e} 0,44 \mathrm{~g} / \mathrm{cm}^{3}\right)$, apresentaram comportamento semelhante, isto é, tiveram ampla variação na sua constante dielétrica, compreendendo intervalo entre 599 e 15 . Essa variação pode ser justificada pelos elevados teores de umidade apresentados quando saturadas, 140 e $115 \%$, respectivamente.

Qualea dinizii, de massa específica média $(0,57$ $\mathrm{g} / \mathrm{cm}^{3}$ ), apresentou variação intermediária, com constante dielétrica variando entre 295 e 17

Tabela 3 - Valores máximos e mínimos da constante dielétrica $(\mathrm{k})$ e teor de umidade.

Table 3 -Maximum and minimum values of dielectric constant $(k)$ and moisture content.

\begin{tabular}{lccccc}
\hline Espécie/k & $\mathrm{k}_{\text {Máx }}$ & $\mathrm{TU}(\%)$ & $\mathrm{k}_{\text {Min }}$ & $\mathrm{TU}(\%)$ & Variação $^{1}$ \\
\hline Brosimum sp. & 606 & 77,00 & 11 & 0,95 & 55,09 \\
Albizia duckeana & 599 & 126,00 & 15 & 2,00 & 39,93 \\
Trattinnickia burserifolia & 589 & 97,00 & 20 & 0,54 & 29,45 \\
Qualea dinizii & 295 & 68,00 & 17 & 1,21 & 17,35 \\
Swartzia laurifolia & 375 & 27,00 & 38 & 0,14 & 9,87 \\
Chamaecristas cleroxylon & 244 & 29,00 & 50 & 2,31 & 4,88 \\
\hline
\end{tabular}

${ }^{1}$ Razão entre os valores máximo e mínimo de constante dielétrica (k).
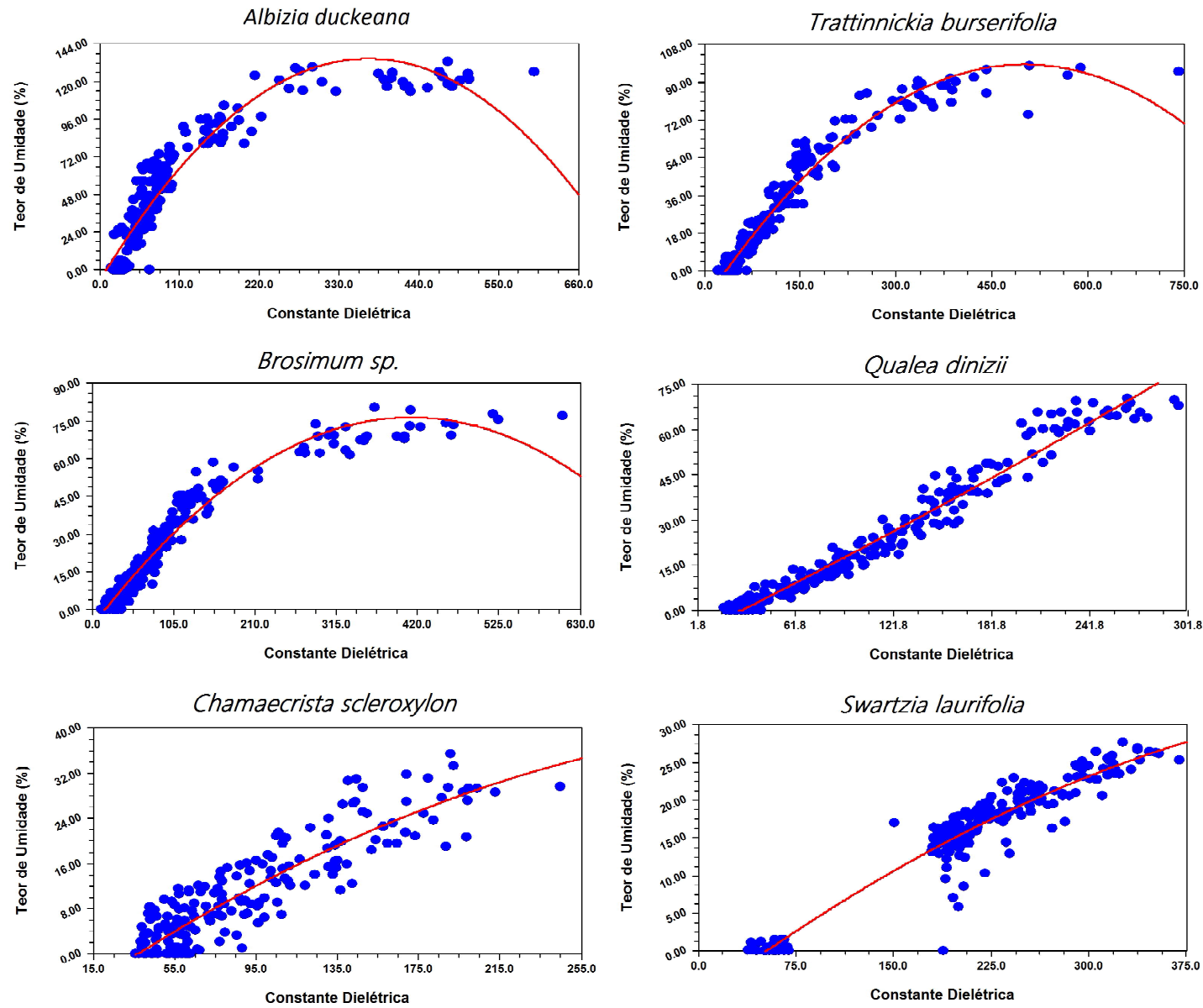

Figura 2 - Curvas de regressão ajustadas para as espécies estudadas.

Figure 2-Regression Curves adjusted for the studied species. 
Chamaecristas cleroxylone e Swartzia laurifolia, de massa específica alta $\left(0,89\right.$ e $\left.1,03 \mathrm{~g} / \mathrm{cm}^{3}\right)$, foram as espécies que apresentaram menor variação na sua constante dielétrica, compreendendo intervalo entre 375 e 38 . Essa variação pode ser justificada pelos baixos teores de umidade quando saturados, 29 e $27 \%$, respectivamente.

\section{DISCUSSÃO}

\subsection{Valores Médios de Constante Dielétrica em Função do Teor de Umidade das Espécies Estudadas}

Os valores de constante dielétrica obtidos compreenderam um intervalo entre 18,44 e 236.493,83, coincidindo com os resultados obtidos por Simpson e Tenwolde et al. (1999), que afirmaram que a constante dielétrica da madeira pode variar de 4 , quando totalmente seca, até próximo de 1.000 .000 , quando a madeira se encontra úmida.

Embora a massa específica exerça influência sobre a propriedade dielétrica da madeira, foi possível observar que as espécies Brosimum sp. e Qualea dinizii, madeiras de massa específica básica de 0,54 e $0,57 \mathrm{~g} / \mathrm{cm}^{3}$ ), apresentaram a maior variação entre os valores máximos e mínimos de constante dielétrica, quando comparados com Trattinnickia burserifolia, de massa específica básica de $0,44 \mathrm{~g} / \mathrm{cm}^{3}$. Esse comportamento foi constatado por Panshin e de Zeeuw et al. (1970), que observaram que a direção da grã e as dimensões das fibras, características intrínsecas a cada espécie, também exercem influência sobre as suas propriedades elétricas, em razão da anatomia.

Aguirre et al. (2010) realizou um estudo avaliando a precisão de medidores de umidade do tipo capacitivo, com madeira de Melia azederach L. (massa específica básica de $0,53 \mathrm{~g} / \mathrm{cm}^{3}$ ), semelhante à Brosimum sp., e constatou que para essa faixa de massa específica os medidores apresentaram resultados satisfatórios. Entretanto, quando realizou a medição do teor de umidade de Apuleia leiocarpa (Vogel) J.F. Macbr. (massa específica básica de $0,80 \mathrm{~g} / \mathrm{cm}^{3}$ ), semelhante a Chamaecristas cleroxylon, os medidores apresentaram resultados diferentes daqueles obtidos pelo método gravimétrico, o que justifica a necessidade de desenvolver equipamentos que estejam calibrados a partir de madeiras tropicais de média e alta massa específica.

Pereira et al. (2008), avaliando medidor elétrico do tipo resistivo com Pinus taeda L. (massa específica básica $0,45 \mathrm{~g} / \mathrm{cm}^{3}$ ) e Eucalyptus saligna $\mathrm{Sm}$. (massa específica básica de $0,43 \mathrm{~g} / \mathrm{cm}^{3}$ ), semelhante a Trattinnickia burserifolia, observou que as medições realizadas pelo equipamento apresentaram valores superiores aos obtidos pelo método gravimétrico. Entretanto, após a análise estatística foi constatada a correlação significativa entre o método gravimétrico e o método resistivo para ambas as espécies, demonstrando a eficiência de utilizar as propriedades elétricas da madeira para determinação do teor de umidade.

\subsection{Curvas e Equações Ajustadas}

$\mathrm{Na}$ faixa de teor de umidade analisada, a constante dielétrica apresentou redução de quase $50 \%$ para todas as espécies, evidenciando a forte influência da umidade abaixo do ponto de saturação das fibras sobre o comportamento da madeira e as suas propriedades elétricas, comportamento que também foi constatado em estudo realizado por Torgovnikov et al. (1993).

\section{CONCLUSÃO}

É possível afirmar que há relação direta entre a constante dielétrica e o teor de umidade e que este pode ser estimado com alto grau de confiabilidade, dado o elevado coeficiente de determinação $\left(\mathrm{R}^{2}\right)$, obtido pelas equações propostas. Entretanto, os resultados são válidos apenas para as espécies avaliadas neste estudo, com amostras de $1 \mathrm{~cm}$ de espessura no sentido tangencial.

Em razão da inexistência de relação direta entre os dados de massa específica e constante dielétrica das espécies estudadas, sugere-se o desenvolvimento de novos estudos para melhor explicar a relação entre essas duas grandezas.

\section{REFERENCIAS}

Aguirre, A.L.M. Avaliação de Medidores Dielétricos de Umidade da Madeira Utilizados na Indústria Moveleira. 2010. Monografia (Graduação em Engenharia Industrial Madereira) - Universidade Federal de Pelotas, Pelotas, 2010.

CRISOSTOMO, M.C. Utilização de um
Capacímetro para Determinar a
Relação entre o Teor de Umidade e
Constante Dielétrica de Seis Espécies 
de Madeiras da Amazônia Durante o Processo de Secagem. 2014. Monografia (Graduação em Engenharia Florestal) -

Universidade de Brasilia, Brasilia, 2014.

NORIMOTO, M.; YAMADA, T. The dieletric properties of wood VI, On the dieletric properties of the chemical constituents of wood and the dieletric anisotropy of wood. Wood Research, v.51, n.12, p.31-43, 1971 .

PANSHIN, A.J.; DE ZEEUW, C. Textbook of wood technology. 4.ed. New York: Mc GrawHill, 1980. 722p.
PEREIRA, J.T.M. Avaliação de Equipamento Medidor Elétrico Resistivo de Umidade da Madeira Utilizando Amostras de Eucalipto e Pinus. 2008. Monografia (Graduação em Engenharia Florestal) - Universidade Federal Rural do Rio de Janeiro, Seropédica, 2008.

SIMPSON, W.; TENWOLDE, A. Wood as an engineering material. Washington: United States Department of Agriculture, Forest Service, 1999. 463p.

TORGOVNIKOV, G.I. Dielectric properties of wood and wood-based materials. New York: State University of New York, 1993. 